\title{
Room Temperature Synthesis of Phosphine Capped Lead Bromide Perovskite Nanocrystals Without Coordinating Solvents
}

\author{
Filip Ambroz, ${ }^{\mathrm{a}}$ Weidong Xu ${ }^{\mathrm{b}}$ Srinivas Gadipelli, ${ }^{\mathrm{d}}$ Dan J. L. Brett, ${ }^{\mathrm{d}}$ Chieh-Ting Lin, ${ }^{\mathrm{b}, \mathrm{c}}$ Claudia Contini, ${ }^{\mathrm{b}}$ \\ Martyn A. McLachlan, ${ }^{c}$ James R. Durrant, ${ }^{c, e}$ Ivan P. Parkin ${ }^{a, *}$ and Thomas J. Macdonald ${ }^{\mathrm{a}, *}$ \\ ${ }^{a}$ Department of Chemistry, University College London 20 Gordon St., London, WC1H 0AJ, UK \\ ${ }^{b}$ Department of Chemistry and Center for Plastic Electronics, Molecular Science Research Hub, \\ Imperial College London, White City Campus, Wood Lane, W12 0BZ, London, UK \\ c Department of Materials and Center for Plastic Electronics, Imperial College London, South \\ Kensington Campus, SW7 2AZ London, UK \\ ${ }^{d}$ Electrochemical Innovation Lab, Department of Chemical Engineering, University College London, \\ WC1E 7JE, London, UK \\ e SPECIFIC, College of Engineering, Swansea University, Bay Campus, Swansea SA1 8EN, UK
}

\begin{abstract}
The room temperature synthesis of perovskite nanocrystals (NCs) is typically achieved by employing a ligand-assisted reprecipitation (LARP) method, which can be handled in air, and its products are comparable to what is obtained using the traditional hot-injection method. However, the LARP method typically requires the use of coordinating polar solvents such as dimethylformamide, that are not appropriate for large-scale production due to toxicity concerns and can also degrade or form defective perovskite NCs. Herein, an amine and oleic acid-free room temperature synthesis of lead bromide perovskite NCs is reported that uses a combination of trioctylphosphine oxide and diisooctylphosphinic acid ligands. This combination of ligands provides a stable platform for the polar-solvent-free synthesis in air of fully inorganic $\mathrm{CsPbBr}_{3}(\mathrm{fwhm} \approx 14 \mathrm{~nm}$, emission $=519 \mathrm{~nm})$ and hybrid organic-inorganic $\mathrm{FAPbBr}_{3}$ (fwhm $\approx 19 \mathrm{~nm}$ ) NCs with photoluminescence emission between $530-535 \mathrm{~nm}$ which is in line with the Rec. 2020 color standards. In addition, it is shown that compared to a traditionally-used ligand combination, phosphine ligands can be easily removed from the surface of the NCs, which is important for the future development of this technology in optoelectronic devices.
\end{abstract}

\section{Introduction}

Lead halide perovskites (LHPs) with the formula $\mathrm{APbX}_{3}\left(\mathrm{~A}=\mathrm{Cs}^{+}, \mathrm{CH}\left(\mathrm{NH}_{2}\right)_{2}{ }^{+}\right.$, etc.; $\left.\mathrm{X}=\mathrm{Cl}, \mathrm{Br}, \mathrm{I}\right)$ are a class of semiconductor materials that have attracted immense research interest in the past decade due to their exceptional photovoltaic and optoelectronic characteristics. ${ }^{[1]}$ They were first reported in $1893 ;{ }^{[2]}$ however, it took more than 100 years to reveal their true potential. ${ }^{[3]}$ While perovskites were initially used for dye-sensitized solar cells, ${ }^{[4]}$ the field of applications where such materials can be implemented has expanded into high-efficiency solid-state solar cells ${ }^{[5]}$ light-emitting diodes (LEDs), ${ }^{[6]}$ photodetectors ${ }^{[7]}$ and many other related technologies. ${ }^{[8]}$ While conventional thin-film LHPs possess many attractive qualities, without passivation they have limited photoluminescence quantum yields (PLQY),${ }^{[9,10]}$ a characteristic that is vital for advancing LEDs and related devices. ${ }^{[1]}$ Therefore, efforts were directed to the synthesis of colloidal perovskite nanocrystals (NCs), which were first reported in 2014 for $\mathrm{CH}_{3} \mathrm{NH}_{3} \mathrm{PbBr}_{3} \mathrm{NCs}{ }^{[12]}$ followed by the preparation of other types of perovskite structures. ${ }^{[13]}$ In addition to higher PLQY value, other benefits of NCs include the possibility to exploit their quantumconfinement which allows for a wide range of tunable emission wavelengths. The latter can also be maneuvered via composition or structuring during the synthesis or via post-synthesis modifications, such as ion exchange or exfoliation. ${ }^{[9,14]}$ Furthermore, NCs have a number of non-conventional properties; for example, the possibility of multiple exciton generation, which in the field of solar cells could result in overcoming the Shockley-Queisser efficiency limit. ${ }^{[15]}$ Recent work in the field has also demonstrated that all inorganic $\mathrm{CsPbI}_{3}$ colloidal NCs stabilize the cubic perovskite crystal phase, 
whereas conventional thin-film $\mathrm{CsPbI}_{3}$ materials relax to the orthorhombic phase at ambient conditions. ${ }^{[16]}$

Compared to chalcogenides, LHP NCs exhibit particular properties that distinguish themselves from the traditional colloidal family of semiconductors. Specifically, they are more ionic in nature and are known to be defect-tolerant. While this does not imply that there are no electronic traps present, in fact, the opposite was shown, ${ }^{[17]}$ but rather they can be bright emitters without the need for extensive surface passivation.

As the performance of applications based on LHP NCs is approaching commercial values, extensive research has been devoted to their efficient synthesis (cost-effective and scalable). Typically, they are synthesized via the hot-injection method $\left(\approx 120^{\circ} \mathrm{C}\right)$ in nonpolar solvents employing a binary ligand system that is composed of aliphatic carboxylic acids and primary amines to solvate the lead salt. Since typically, the hot-injection approach requires an inert atmosphere, neither is particularly cost-effective or industrially feasible, efforts have been made aiming for alternative energy efficient methods, such as low temperature approaches and ambient air conditions. ${ }^{[18]}$ To complement this, the ligand-assisted reprecipitation (LARP) technique is commonly employed where a DMF solution of precursor salts is rapidly injected into a vigorously stirred antisolvent media. However, the technique has certain drawbacks that limit the industrial-scale utilization. Particularly, polar coordination solvents such as DMF are not that appropriate for industrial-scale synthesis due to toxicity concerns. In addition, DMF can easily degrade and even dissolve perovskite NCs where the precursor-polar solvent interactions have a strong influence on the formation of defective perovskite NCs. ${ }^{[18-22]}$ Moreover, high temperature activation is required to remove DMF molecules, further highlighting the disadvantage of such polar solvent use. Therefore, it is highly desired to develop new synthesis methods that are energy efficient and do not require the use of polar solvents. While efforts have been made to eliminate the use of polar solvents, they typically still require oleic acid/oleylamine (OA/OAm) in a combination with lengthy probe sonication or high temperature precursor heating steps to achieve good quality NCs, both of which require a lot of energy. ${ }^{[18,23,24]}$ In addition, probe sonication is not scalable, and this is a major concern preventing such approaches from being industrially feasible.

Herein, we demonstrate a simple route to synthesize fully inorganic and organic-inorganic perovskite $\mathrm{NCs}$ at RT in air, without the use of polar solvents, and thus favoring industrial scale up. For this purpose, traditionally used ligands were replaced with a phosphine-based alternative using industrially compatible solvents, such as octadecene (ODE). Our approach demonstrates a straightforward synthesis of fully inorganic $\left(\mathrm{CsPBr}_{3}\right)$ and organic-inorganic hybrid $\left(\mathrm{FAPbBr}_{3}, \mathrm{FA}=\mathrm{CH}\left(\mathrm{NH}_{2}\right)_{2}{ }^{+}\right)$perovskite $\mathrm{NCs}$ to exhibit high quality crystallinity and cubic phase structure. Specifically, $\mathrm{FAPbBr}_{3} \mathrm{NCs}$ show to exhibit light emission with a narrow full-width at half-maximum ( $\mathrm{fwhm}$ ) value of $\approx 19 \mathrm{~nm}$ in the wavelength range between $530-535 \mathrm{~nm}$ which is a desirable milestone according to Rec. 2020 color standards. ${ }^{[25]}$ Using spectroscopy techniques, the way in which the ligands interact with NC surfaces is investigated and we show that the phosphine ligands are dynamically bound to the surface via a $\mathrm{Pb}-\mathrm{O}$ $\mathrm{P}$ bond. Furthermore, one of the main drawbacks of NCs is the undesirable presence of non-conductive ligands that can severely influence the charge transport capabilities of electrons and holes when they are used in optoelectronics. Herein, it is shown that compared to a widely used ligand combination of $\mathrm{OA}$ and OAm, the phosphine-based alternative presented in this work can be easier to remove from the NC surface thus offering high-quality NCs with a lower content of organics, which is vital for their application in optoelectronics.

\section{Results and Discussion}

\section{Nanocrystal Synthesis and Their Structural Properties.}

In this synthesis, the traditional OA/OAm ligand couple is replaced with a phosphine-based combination of trioctylphosphine oxide (TOPO) and diisooctylphosphinic acid (TMPPA). In a typical $\mathrm{NC}$ synthesis adapted from Protesescu et al., ${ }^{[13]} \mathrm{ODE}$ solvent is used together with chelates such as fatty acid (oleic acid) to dissolve an $\mathrm{A}$ - site cation source of a perovskite crystal structure ( $\mathrm{ABX}_{3}$ type). $\mathrm{A}$ separate solution for the $\mathrm{B}$ and $\mathrm{X}_{3}$ - sites is also needed which normally consists of $\mathrm{PbX}_{2}$ in $\mathrm{ODE}$ with 
$\mathrm{OA}$ and $\mathrm{OAm}$ where both ligands are required in order to dissolve $\mathrm{PbX}_{2}$. To synthesize NCs, both precursors have to be dissolved using the typical ligand couples of OA/OAm, which is only viable at elevated temperatures of $\approx 110^{\circ} \mathrm{C}$. While the absence of an amine can decrease the reaction temperature $\left(\approx 75{ }^{\circ} \mathrm{C}\right)$, elevated temperatures $\left(\approx 120{ }^{\circ} \mathrm{C}\right)$ are required to dissolve $\mathrm{Cs}$ and $\mathrm{Pb}$ precursors. ${ }^{[26]} \mathrm{By}$ replacing OA with TMPPA in the A - site precursor solution, we were able to solubilize both FA (formamidinium)-acetate salt and $\mathrm{Cs}_{2} \mathrm{CO}_{3}$ (cesium carbonate) in ODE at RT. Furthermore, the same is done for the $\mathrm{BX}_{3}$ precursor solution in ODE where TMPPA was used in a combination with TOPO. Again, the Pb salt was successfully dissolved and formed a clear solution. It should be noted that TOPO alone can also solubilize the $\mathrm{Pb}$ salt in ODE without the presence of TMPPA under the same conditions; however, syntheses in this study were always performed using a combination of both since the presence of both TOPO and TMPPA produced much higher quality NCs. Based on the solubility of the precursor solutions in the ligand couples, Cs and FA-based perovskite NCs were synthesized via a single-step injection at RT in air - overriding the use of a Schlenk line and high temperature conditions. Videos 1 and 2 in the Supporting Information (SI) show the rapid synthesis of perovskite NCs with different cations, Cs and FA. Such ionic metathesis reactions occur very quickly; however, the rate of nucleation and growth kinetics for different cations are dissimilar. Specifically, the initially colorless solution of $\mathrm{Pb}$ salt turns immediately colored when FA-acetate is injected, indicating the instantaneous formation of FA-cation NCs, as opposed to the delayed nucleation upon the injection of $\mathrm{Cs}_{2} \mathrm{CO}_{3}$, which leads to the formation of Cs-cation type of NCs. Previous studies have suggested strong correlation between the nucleation rate and growth stages of NCs with the ligands used during the synthesis. ${ }^{[27]}$ Since the synthesis used the same ligands for both types of NCs, the slow nucleation of $\mathrm{CsPBr}_{3}$ can be attributed to the smaller cation size compared to FA. The ease, by which such highly crystalline compounds were synthesized at RT conditions, where no protective atmosphere was required, is a result of their ionic bonding. They were clearly identified and labelled as $\mathrm{Phos}_{-} \mathrm{FAPbBr}_{3}$ (phosphinic $\mathrm{FAPbBr}_{3}$ ) or Phos$\mathrm{CsPbBr}_{3}$ (phosphinic $\mathrm{CsPbBr}_{3}$ ) NCs and resulted in cube-like structures. Figure 1a, b illustrates a TEM image of Phos-CsPbBr 3 NCs with an average edge length of $\approx 14 \mathrm{~nm}$ and standard size deviation $(\sigma)$ of $\approx 12 \%$. High crystallinity can be observed from the HR-TEM image, as confirmed with a characteristic lattice spacing of $\approx 5.8 \AA$, which corresponds to the (100) crystal plane of the cubic perovskite structure (Figure 1c). Notably, as opposed to Phos-CsPbBr 3 , $\mathrm{Phos}_{-} \mathrm{FAPbBr}_{3} \mathrm{NCs}$ were difficult to image since the NCs were sensitive to the TEM beam. However, Figure S1 shows the TEM image of Phos-FAPbBr NCs with an average edge length of $\approx 38 \mathrm{~nm}(\sigma \approx 16 \%)$ and a lattice spacing of $\approx 5.8 \AA$. Furthermore, the crystal structure of the NCs has been additionally characterized by XRD (Figure 1d). Pure cubic perovskite phase for both types of NCs is displayed, matching well with the Ref-FAPbBr 3 (for JPCDS

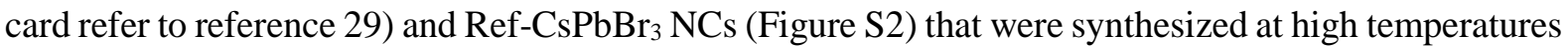
following the established procedure by Protesescu et al. ${ }^{[13]}$ It should be noted that in this work, OA/OAm


that the XRD crystal planes peak intensities for both types of phosphinic NCs are enhanced and better defined compared to the reference $\mathrm{NCs}^{[13]}$ (Figure S2) indicating improved crystallinity. 




(d)

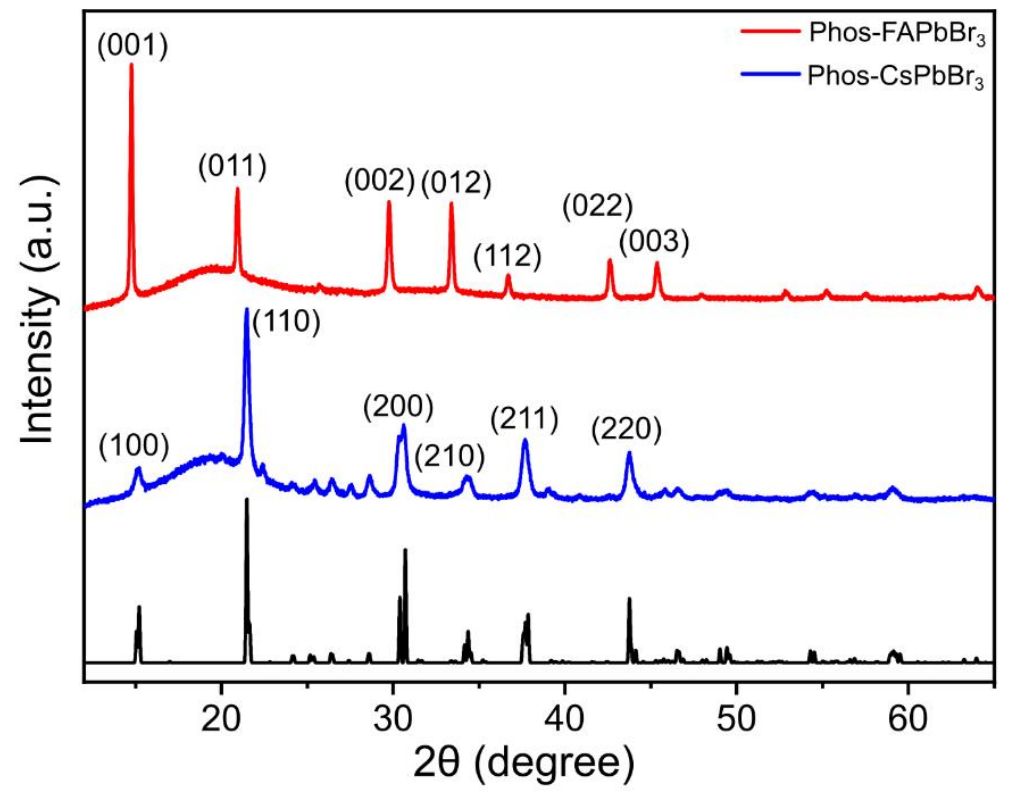

Figure 1. (a, b) TEM images of Phos- $\mathrm{CsPbBr}_{3} \mathrm{NCs}$ and (c) corresponding HR-TEM image confirming high crystallinity and associated lattice space. (d) XRD patterns for both types of phosphinic NCs. Standard $\mathrm{CsPbBr}_{3}$ phase is shown in black (bottom). For standard $\mathrm{FAPbBr}_{3}$ phase, refer to reference 29.

\section{Optical properties}

The optical UV-Vis absorption of phosphinic NCs reveals a single peak at the first excitonic transition for $\mathrm{Phos}_{-} \mathrm{FAPbBr}_{3}$ (Figure 2a) while for $\mathrm{Phos}_{-\mathrm{CsPbBr}}$ two defined peaks are observed, where the second electronic transition appeared at $\approx 375 \mathrm{~nm}$ (Figure $2 \mathrm{~b}$ ). Such discrete electronic transitions can be observed in the absorption spectra of high-quality nanocrystals as reported by Norris Bawendi. ${ }^{[28]}$ While this second electronic transition has also been observed in earlier work on $\mathrm{CsPbBr}_{3} \mathrm{NCs}$ by Protesescu et al ${ }^{[29]}$, it is absent in reports on $\mathrm{FAPbBr}_{3} \mathrm{NCs}^{\left[{ }^{[30]}\right.}$ also consistent with our results. We postulate that the large size of our Phos-FAPbBr 3 NCs limits the possibility for other electronic transitions to be well defined in the absorption spectra. ${ }^{[31]}$ Moreover, near monodisperse size distribution (Figure 1a) for Phos-CsPbBr $3{ }_{3} \mathrm{NCs}$ can also be attributed to the second electronic transition to be more clearly observed. ${ }^{[32]}$ Their emission peaks were shown to be very sharp with narrow emission line widths of $\approx 19 \mathrm{~nm}$ for $\mathrm{Phos}-\mathrm{FAPbBr}_{3}$ and $\approx 14 \mathrm{~nm}$ for $\mathrm{Phos}-\mathrm{CsPbBr}_{3} \mathrm{NCs}$ which is comparable to the energy intense hot-injection method. ${ }^{[13,30]}$ The cation size difference influenced the position of the 
emission peaks. Specifically, compared to Phos-FAPbBr 3 with an emission peak at $532 \mathrm{~nm}$, Phos$\mathrm{CsPbBr}_{3} \mathrm{NCs}$ were shown to be blue shifted (emission peak at $519 \mathrm{~nm}$ ) while having the same halogen composition. Furthermore, the narrow emission peak at $532 \mathrm{~nm}$ of Phos-FAPbBr ${ }_{3} \mathrm{NCs}$ is in line with the three primary emission peaks for displays and for the wide-gamut colorimetry (467, 532 and 630 $\mathrm{nm}$ ) as reported by the International Commission on Illumination (CIE) and the International Telecommunication Union (ITU) rendering them as a promising candidate for light emission applications. ${ }^{[33]}$ To investigate the PL tuning of phosphinic NCs without altering their halide composition, syntheses were performed under various reaction temperatures ranging from RT up to 130 ${ }^{\circ} \mathrm{C}$. It should be noted that before the injection, both precursor solutions were kept at the same temperatures.

Quantum confinement influences PL emission where the bandgap typically increases as the size of the NCs decrease, which is a common phenomenon observed in perovskite NCs. While the reference NCs can be single compositionally tuned in the visible region in the range of $\approx 65 \mathrm{~nm}$ for $\mathrm{FAPbBr}_{3}$ and $\approx 35$ $\mathrm{nm}$ for $\mathrm{CsPBBr}_{3},{ }^{[13,30]}$ the results show that phosphinic NCs at different synthesis temperatures essentially did not exhibit any PL peak tuning at all (Figure S3). Such a phenomenon can be related to their sizes that were significantly larger than the exciton Bohr radius, resulting in no quantum confinement. ${ }^{[9]}$ However, it was observed that their PLQYs varied as a function of low- or hightemperature conditions which is illustrated in Figure $2 \mathrm{c}$ and $2 \mathrm{~d}$ together with the corresponding fluorescence photographs (Figure 2e and 2f, respectively. See Figure S4 for photographs without the excitation). By comparing the colloidal solutions of both phosphinic NCs, an orange colloidal solution formed for Phos-FAPbBr 3 while it turned lime green for $\mathrm{Phos}_{-} \mathrm{Cs} \mathrm{PbBr}_{3}$. The trends indicate that the hot-injection approach negatively influences the fluorescence of NCs when phosphinic based ligand couples are used. Compared to the high-temperature synthesis, RT conditions result in higher PLQY values, indicating that the hot-injection approach can act detrimentally to obtain high fluorescence in phosphinic NCs. Since PLQY is directly related to the surface passivation, it is postulated that high synthesis temperatures lead to the creation of in-gap trap states that could act as nonradiative recombination centers. ${ }^{[19]}$

Different concentrations of the A-site precursor solution can influence the surface coverage characteristics of NCs. ${ }^{[19]}$ Therefore, PLQY as a function of the FA-acetate concentration is reported in Figure S5. It was determined that the concentration increase had a detrimental impact on the PLQY where the most optimal concentration was found to be $0.1 \mathrm{M}$. It should be noted that the measurements were performed only for FA-acetate which is at different concentrations and under RT conditions easier to dissolve compared to $\mathrm{CsCO}_{3}$. Furthermore, the concentration influence of TMPPA in the $\mathrm{BX}_{3}$ precursor solution was also monitored for both types of NCs where no trends in the PLQY values were observed. Compared to the synthesis with OA/OAm ligands and the PLQYs reported in the literature, ${ }^{[34]}$ phosphinic NCs were shown to be less photoluminescent and the phenomenon can be related to the interactions between the surface states and the coordinated ligands. However, such results are not surprising where previous work by Moon et al. ${ }^{[35]}$ demonstrates how $\mathrm{MgO}$ NCs exhibit a $50 \%$ lower PLQY when TOPO was used as a coordinating ligand compared to benzyl ether.

The time-resolved PL traces from solutions of phosphinic NCs were measured and fitted with triexponential decay; while for the reference NCs, bi-exponential decay fitting was used which is illustrated in Figure 2g-h and the details of decay lifetime values are given in Table S1 and S2. The absence of significant decay kinetics in $<1 \mathrm{~ns}$ is indicative of the absence of rapid charge trapping into non-radiative trap sites in the NCs. ${ }^{[36]}$ In the literature, there are many reports of a size effect of NCs where emission decay lifetimes increase with bigger size, which is related to slower bimolecular recombination due to longer charge diffusion lengths. ${ }^{[36,37]}$ Therefore, larger NCs will exhibit longer lifetimes compared to their smaller counterparts. In this study, results are compared for TCSPC of RT synthesized phosphinic and reference $\mathrm{FAPbBr}_{3}$ and $\mathrm{CsPbr}_{3} \mathrm{NCs}$ (Figure $2 \mathrm{~g}$ and $2 \mathrm{~h}$ ). It was observed that the former type exhibit longer average emission decay lifetimes which are attributed to their bigger sizes, as calculated via the Scherrer equation (see Figure 1 and S1 for XRD patterns). The average crystallite size of $\mathrm{Phos}_{-} \mathrm{FAPbBr}_{3}$ was $\approx 2.4$ times bigger, while $\mathrm{Phos}_{-\mathrm{Cs}} \mathrm{PbBr}_{3} \mathrm{NCs}$ exhibit on average 
$\approx 3.1$ times bigger size in comparison with their respective references. Such a size difference resulted in the average lifetime of the former type to be $\approx 28.5 \mathrm{~ns}$, while the lifetime of the latter type was $\approx 34.8 \mathrm{~ns}$. On the other hand, Ref-FAPbBr 3 and Ref-CsPbBr 3 NCs, exhibited shorter lifetimes of $\approx 12.2$ and $\approx 9.7$ ns, respectively. While reference NCs exhibited higher PLQYs compared to phosphinic NCs, which typically results in longer lifetimes, our results indicate that such a large size difference has a bigger influence on the emission lifetime characteristics compared to PLQYs. Phosphinic NCs were prepared using off-the-shelf chemicals in air and without the need of heating which together with their long emission lifetimes renders them as a promising alternative for the use in optoelectronic applications.

(a)

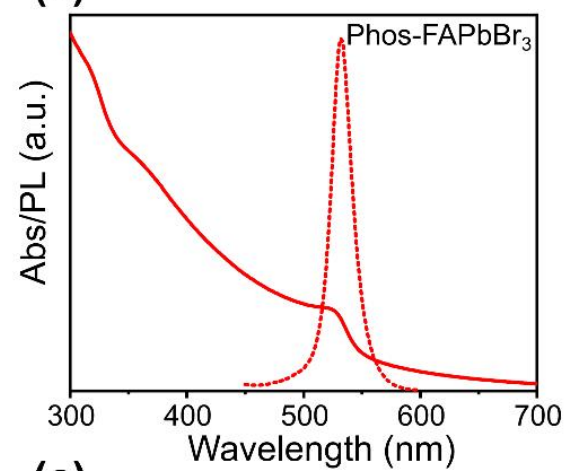

(c)

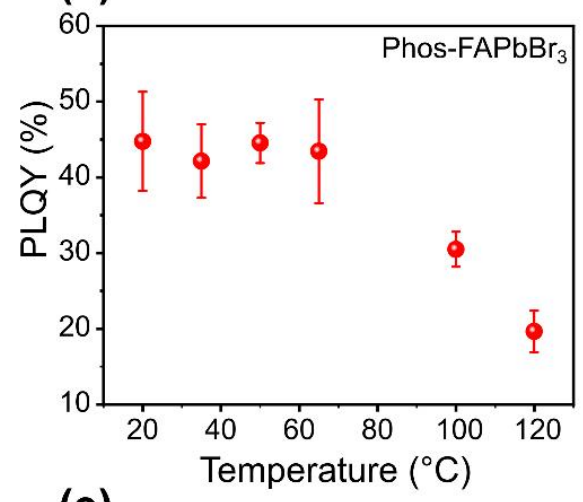

(e)

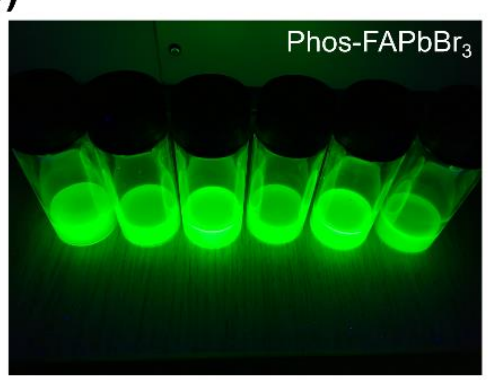

(g)

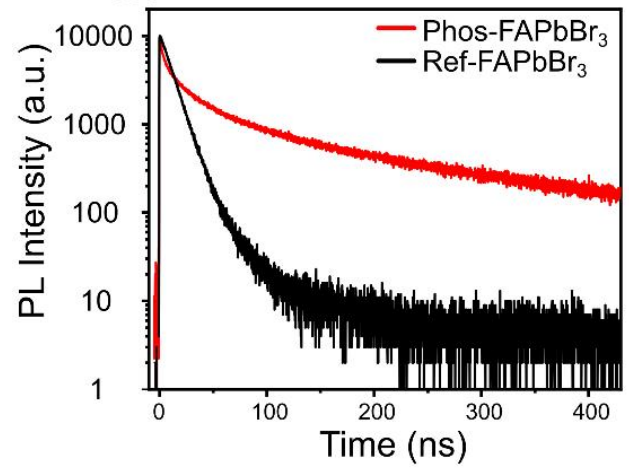

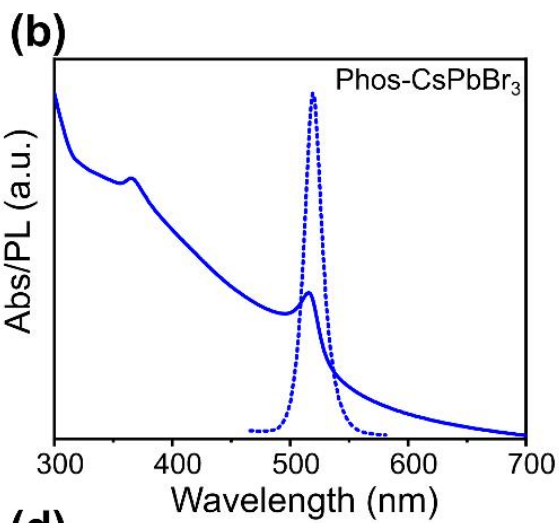

(d)

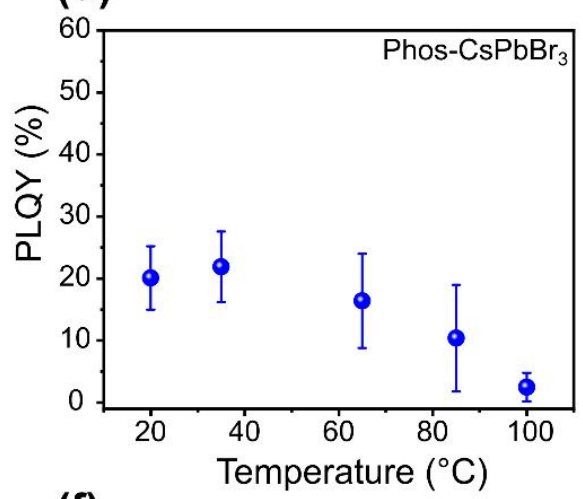

(f)

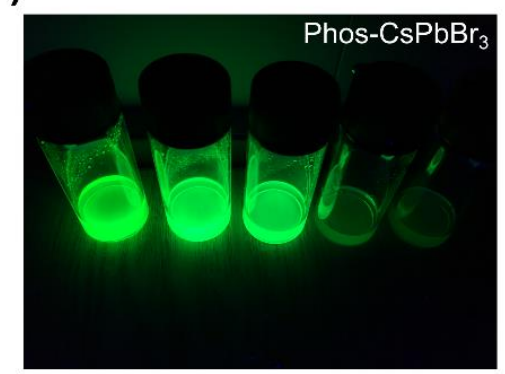

(h)

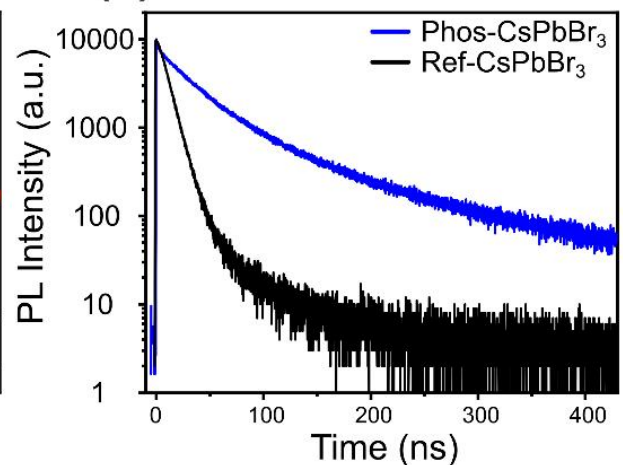


Figure 2. Absorbance (solid line) and photoluminescence (dotted line) spectra of (a) $\mathrm{Phos}_{-} \mathrm{FAPbBr}$ and (b) Phos-CsPbBr 3 NCs synthesized at RT. PLQYs and photographs of a series of (c, e) Phos$\mathrm{FAPbBr}_{3}$ and $(\mathrm{d}, \mathrm{f}) \mathrm{Phos}_{-\mathrm{CsPbBr}} \mathrm{NCs}$ synthesized at different reaction temperatures and under UV light $(\lambda=365 \mathrm{~nm})$ excitation. PL decay dynamics of (g) Phos-FAPbBr 3 and (h) Phos-CsPbBr 3 NCs colloidal solutions.

\section{Nanocrystal-Ligand Composition}

X-ray photoelectron spectroscopy (XPS) was carried out to identify elements present on the surface of phosphinic NCs. Elements ( $, \mathrm{P}, \mathrm{O}, \mathrm{N}, \mathrm{Cs}, \mathrm{Pb}$ and $\mathrm{Br}$ ) associated to phosphinic ligands and the perovskite crystal structure were identified in Figure 3(a-d) and Figure S6 and S7. Furthermore, P 2p and $\mathrm{O} 1 \mathrm{~s}$ spectra were used to investigate surface ligand interactions with the NCs core. No peaks appeared that correspond to elemental phosphorous (range between $129-131 \mathrm{eV}$ ); however, it shows a broad band at $131-135 \mathrm{eV}$ associated for higher oxidation state (Figure 3a and 3c). This is deconvoluted into peaks that correspond to different $\mathrm{P}$ bonding. ${ }^{[38]}$ The exact nature of $\mathrm{P}$ bonding is identified from the deconvoluted $\mathrm{O} 1 \mathrm{~s}$ spectra (Figure $3 \mathrm{~b}$ and $3 \mathrm{~d}$ ). The peak at lower binding energy $\left(\approx 532 \mathrm{eV}\right.$ for $\mathrm{CsPbBr}_{3}$ and $\approx 531 \mathrm{eV}$ for $\left.\mathrm{FAPbBr}_{3}\right)$ was assigned to the $\mathrm{O}$ that did not participate in the bonding $(\mathrm{P}=\mathrm{O})$, while the peak at higher binding energy $\left(\approx 533 \mathrm{eV}\right.$ for $\mathrm{CsPbBr}_{3}$ and $\approx 532 \mathrm{eV}$ for $\mathrm{FAPbBr}_{3}$ ) was assigned to the $\mathrm{O}$ that was part of the $\mathrm{Pb}-\mathrm{O}-\mathrm{P}$ bond. ${ }^{[38,39]}$ The results indicate that the $\mathrm{P}=\mathrm{O}$ group of the phosphinic ligands act as a center for ligand binding on NCs surfaces which leads in the formation of a bond with $\mathrm{Pb}$ element of the perovskite crystal structure. To gain further insights on such surface interactions, FTIR spectroscopy was implemented where the findings are discussed in the sections below.

Figure $3 \mathrm{e}$ and $3 \mathrm{f}$ illustrates the elemental compositions on the surface of Phos-FAPbBr $3 \mathrm{NCs}$ at different synthesis temperatures. We see that with increasing the synthesis temperature, $\mathrm{N}: \mathrm{Pb}$ and $\mathrm{Br}: \mathrm{Pb}$ atomic ratios increase, which effect relates to the decreasing composition of $\mathrm{Pb}$. Moreover, by comparing $\mathrm{Pb}: \mathrm{P}$ atomic ratios the same trend can be observed where as opposed to the RT synthesis $(\mathrm{Pb}: \mathrm{P}=1: 2.9-3.9)$ the atomic ratios for the highest temperature notably increased $(\approx \mathrm{Pb}: \mathrm{P}=1: 20)$. On the other hand, for Phos-CsPbBr 3 NCs, no difference in the $\mathrm{Br}: \mathrm{Pb}$ atomic ratios were noted (Figure S8) while the same trends as noted before for $\mathrm{FAPbBr}_{3}$ were observed for the $\mathrm{Pb}: \mathrm{P}$ atomic ratios. The $\mathrm{Pb}: \mathrm{P}$ atomic ratios for both types of NCs revealed that the ligand interactions with the NCs cores and thus the surface elemental compositions are similarly as PLQY values also influenced by the synthesis temperatures.

To monitor the presence of $\mathrm{P}$ ligands on the surface of NCs after the antisolvent washing (for purification details see experimental section in $\mathrm{SI}$, $\mathrm{Pb}: \mathrm{P}$ atomic ratios were determined for Phos$\mathrm{FAPbBr}_{3}(\mathrm{~Pb}: \mathrm{P}=1.2-1.7: 1)$ and $\mathrm{Phos}_{-} \mathrm{CsPbBr}_{3}(\mathrm{~Pb}: \mathrm{P}=1.8-2.2: 1) \mathrm{NCs}$, respectively. Here, the amount of $\mathrm{Pb}$ was shown to exceed that of $\mathrm{P}$ which is the opposite to before. Such results indicate that a significant amount of ligands was removed from the $\mathrm{NC}$ surfaces, illustrating a weak $\mathrm{Pb}-\mathrm{O}-\mathrm{P}$ bonding to the $\mathrm{NC}$ core. 
(a)

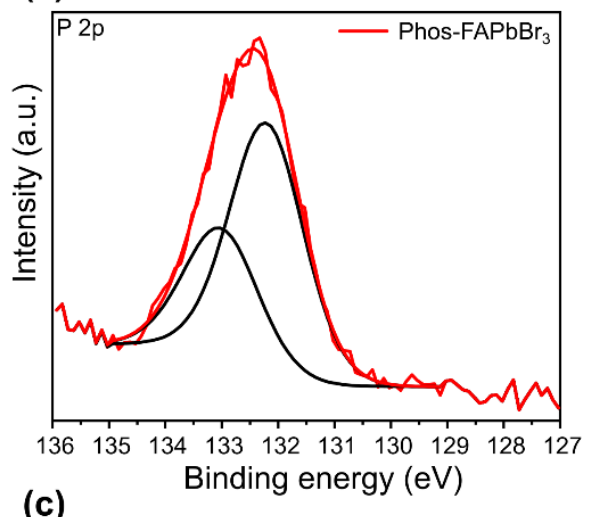

(c)
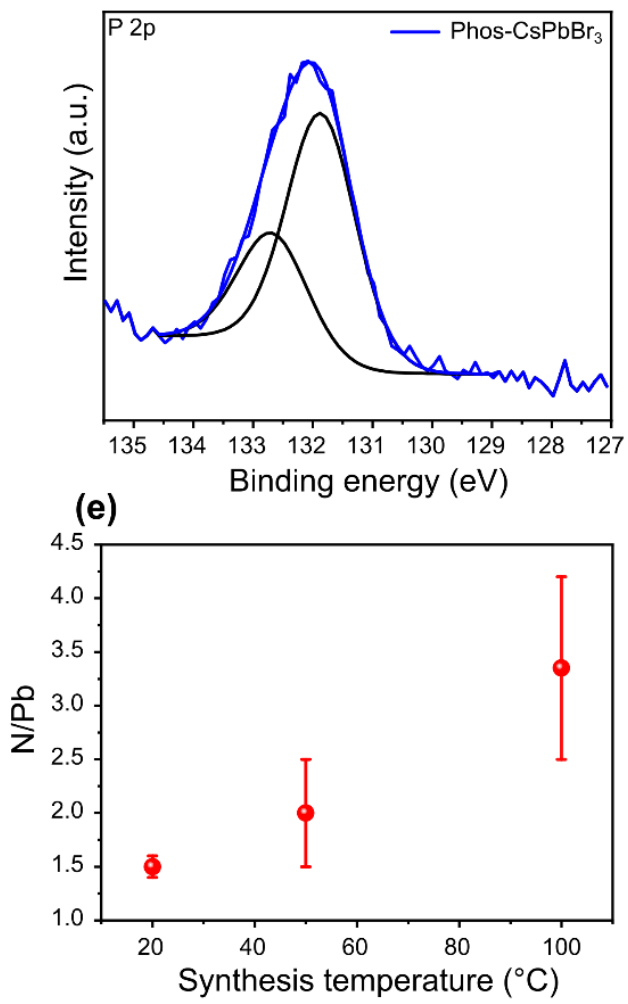

(b)

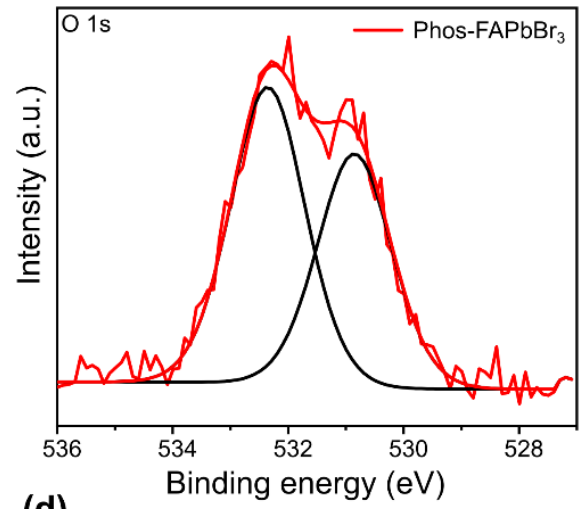

(d)

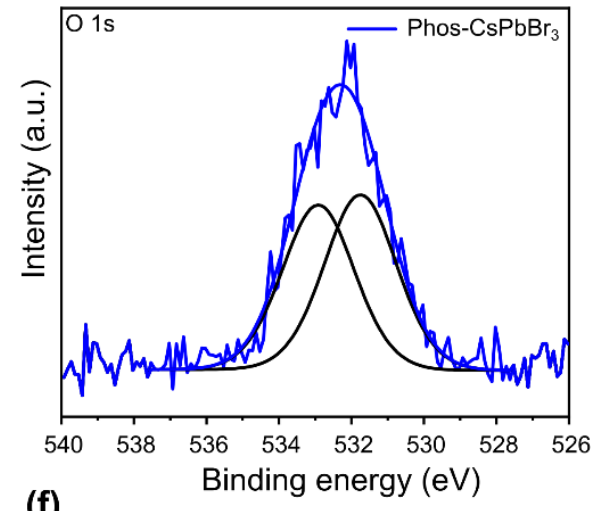

(f)

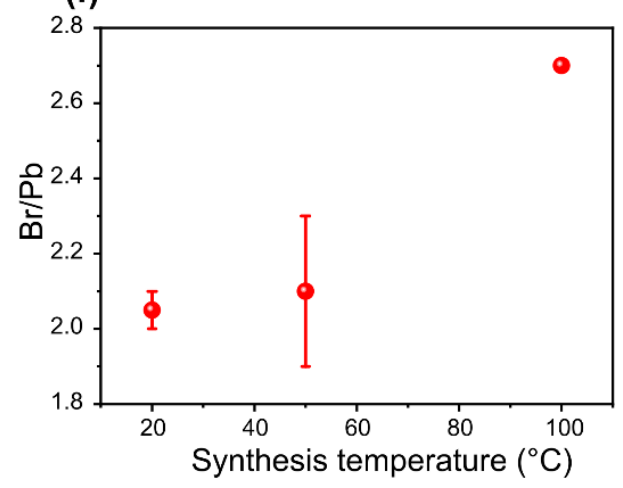

Figure 3. The core level (a) P 2p and (b) O 1s XPS spectra for Phos-FAPbBr $\mathrm{NCS}_{3}$ and (c) P 2p and (d) $\mathrm{O} 1 \mathrm{~s}$ XPS spectra for Phos-CsPbBr 3 NCs. (e) N:Pb and (f) $\mathrm{Br}: \mathrm{Pb}$ atomic ratios (as determined by XPS) for a series of $\mathrm{Phos}_{-\mathrm{FAPbBr}} \mathrm{NCs}$ synthesized at different reaction temperatures.

\section{NC Surface-Ligand Interactions}

Fourier-transform infrared (FTIR) spectroscopy was used to characterize the surface of NCs and gain insights into structure surface-ligand interactions of phosphinic based surfactants (Figure $4 \mathrm{a}$ and $4 \mathrm{~b}$ ). The FTIR spectra of TOPO and TMPPA ligands are illustrated in Figure S9 with the corresponding structural formulas in Figure S10. Spectral assignments of the main signals are given in Table S3 and S4. Compared to the FTIR spectrum of both ligands, the intensity and position of the $\mathrm{P}=\mathrm{O}$ stretching vibration has notably changed for NCs (Figure 4a). As noted from the studies of transition metal complexes, ${ }^{[40]}$ the formation of the coordination complexes from the $\mathrm{O}$ atom of the $\mathrm{P}=\mathrm{O}$ stretching group of $\mathrm{R}_{3} \mathrm{PO}$ molecules (TOPO - $\left.\left[\mathrm{CH}_{3}\left(\mathrm{CH}_{2}\right)_{7}\right]_{3}\right)$ results in the lower wavenumber. This peak shift indicates TOPO binding to NCs surfaces. ${ }^{[35]}$ The origin of the shift can be attributed to a transfer of electron density from $\mathrm{P}$ to $\mathrm{O}$, resulting in the frequency decrease of the $\mathrm{P}=\mathrm{O}$ group and lower absorption of infrared radiation. ${ }^{[41]}$ Here, the $\mathrm{P}=\mathrm{O}$ stretching peak has broadened and red-shifted $\left(1120 \mathrm{~cm}^{-1}\right)$ compared to the corresponding vibrations for pure ligands. Therefore, this indicated that the $\mathrm{P}=\mathrm{O}$ bond 
of the ligand was broken to produce a metal complex, thus forming the $\mathrm{Pb}-\mathrm{O}-\mathrm{P}$ bond ${ }^{[42]} \mathrm{A}$ similar alteration of the $\mathrm{P}=\mathrm{O}$ peak where TOPO was used as a ligand has been observed previously for $\mathrm{CdSe}$ NCs where they were referred as TOPO-capped ${ }^{[43,44]}$ and CdS NCs. ${ }^{[40]}$

Furthermore, the role of TMPPA ligand on the surface species of NCs was interpreted based on the P$\mathrm{OH}$ group. Compared to the spectrum of pure TMPPA (Figure S9), two peaks that were previously attributed to the $\mathrm{P}-\mathrm{OH}$ stretching and bending (Table S4) shifted to smaller frequencies on the spectrum of NCs. Specifically, P-OH stretching is present at $\approx 1642 \mathrm{~cm}^{-1}$ while the $\mathrm{P}-\mathrm{OH}$ bending mode is at 908 $\mathrm{cm}^{-1}$, as illustrated in Figure 4a. The interaction of metal species (e.g. Cu) with TMPPA ligand was reported elsewhere ${ }^{[45]}$ where it resulted in a shift of peaks to smaller frequencies. Therefore, the results suggest that the shift can be attributed to the interaction with $\mathrm{Pb}$. Based on the positions of $\mathrm{C}-\mathrm{H}$ absorption bands ( $<3000 \mathrm{~cm}^{-1}$, see Figure $\mathrm{S} 11$ for a closer view), it was determined that ligands on the surface of NCs contain hydrogens bonded to $\mathrm{sp}^{3}$ carbons (see SI for further discussion). As illustrated in Figure 4a and discussed herein, the absorption bands related to the surface ligands were not influenced by the inorganic/organic cation group of NCs. However, compared to $\mathrm{CsPbr}_{3}$ there are additional signals on the spectrum of $\mathrm{FAPbBr}_{3} \mathrm{NCs}$ which can be identified as absorption bands of the additional bonds present in formamidinium (see SI for further discussion).

FTIR technique was also used to investigate the binding strength of ligands on the surface of NCs after the antisolvent washing. Phos-FAPbBr 3 NCs (Figure 4b) showed an immense decrease of peaks corresponding to the $-\mathrm{CH}_{3}$ and $-\mathrm{CH}_{2}$ absorption bands $\left(<3000 \mathrm{~cm}^{-1}\right.$ and the band at $\left.\approx 1462 \mathrm{~cm}^{-1}\right)$. In addition, the peak at $\approx 720 \mathrm{~cm}^{-1}$ almost entirely disappeared, indicating that the amount of organic species with long chain methylene groups has significantly decreased. The same is also seen for P-OH stretching and bending modes, where the former absorption band completely disappeared, as opposed to the bending mode where a weak peak can still be noticed. Considering that all the signals of phosphinic based ligands are substantially reduced, this indicates that their binding strength on the surface of NCs is weak. On the other hand, for Phos-CsPbBr 3 NCs, the intensity of peaks assigned to the organic ligands moderately reduced after the purification (Figure S13 and experimental section for purification details). However, compared to the Phos-FAPbBr 3 NCs (Figure 4b) all the signals were still substantial, indicating that lower amounts of ligands were removed from their surfaces.

To investigate the amount of organics that can be removed during the purification, thermogravimetric analysis (TGA) was performed, where both, namely reference and phosphinic NCs, were purified (experimental section in SI) with the same ratio of antisolvents, i.e. acetonitrile was used for Phos$\mathrm{FAPbBr}_{3}$ and methyl acetate for Phos- $\mathrm{CsPbr}_{3} \mathrm{NCs}$. Figure $4 \mathrm{c}$ and $4 \mathrm{~d}$ show the representative TGA curves. A clear difference in the mass-loss behavior can be observed for both types, illustrating that phosphinic ligands were more successfully removed from their surfaces. Only the organic compounds present are expected to be decomposed below $\approx 450{ }^{\circ} \mathrm{C}$, and the major mass-loss above $550{ }^{\circ} \mathrm{C}$ is attributed to the actual perovskite structure decomposition of NCs. Accordingly, both reference samples lost more weight compared to the phosphinic samples (12.5 mass\% for $\mathrm{FAPbBr}_{3}$ and 12.9 mass\% for $\mathrm{Cs} \mathrm{PbBr}_{3}$ ). This suggests that the binding strength of OA/OAm species on the surface of NCs is more robust toward the polar solvent wash compared to the bonding interactions of phosphinic based ligands. Such findings should be informative for further studies of ligand exchange of perovskite NCs and for their surface modifications to improve charge transport characteristics that are critical for optoelectronic applications. ${ }^{[4]}$ Together with the FTIR, these results support our earlier observation where improved crystallinity was observed in the XRD spectra for the phosphine based NCs in comparison to the reference NCs containing OA/OAm. 
(a)
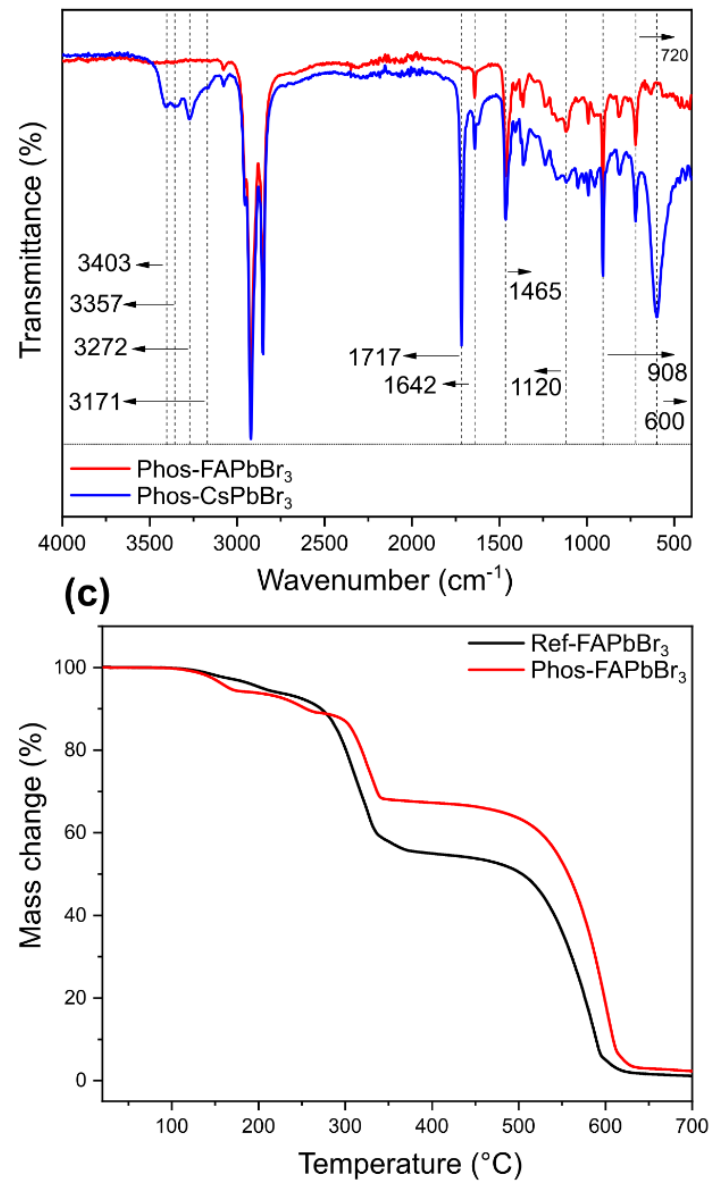

(b)
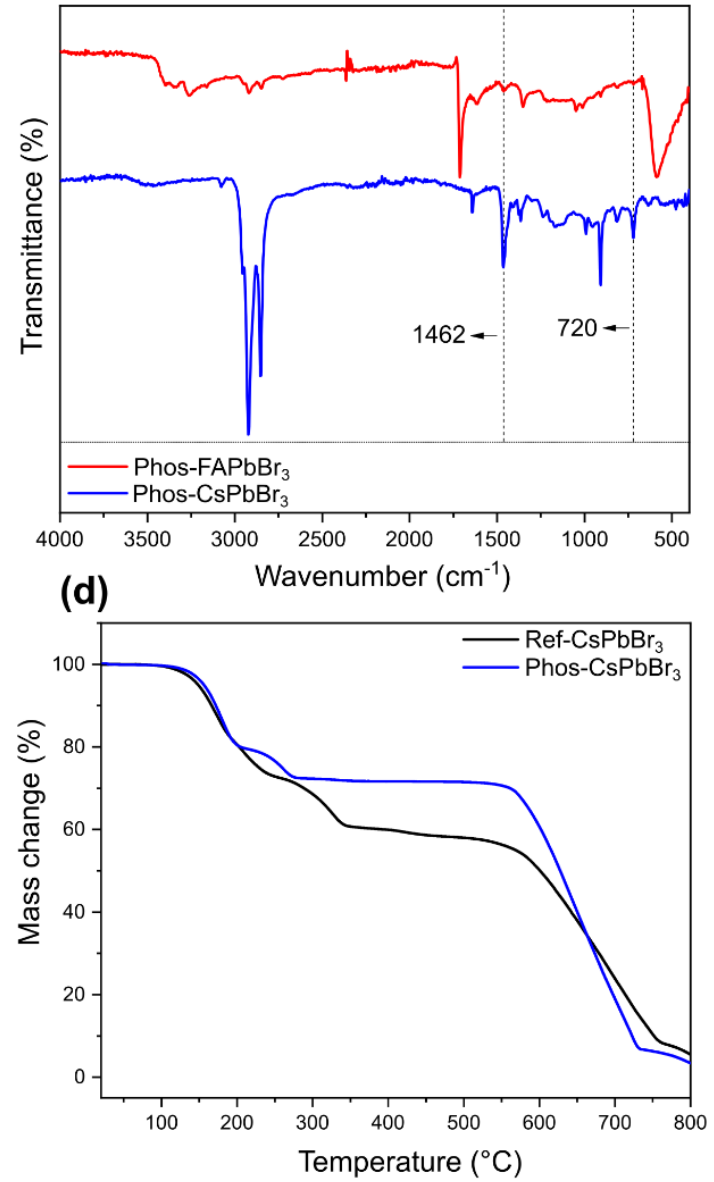

Figure 4. FTIR spectra of (a) unpurified and (b) purified $\mathrm{Phos}_{-} \mathrm{FAPbBr}_{3}$ (red lines) and $\mathrm{Phos}-\mathrm{CsPbBr} 3$ (blue lines) NCs. TGA curves of purified (c) $\mathrm{FAPbBr}_{3}$ and (d) $\mathrm{CsPbBr}_{3} \mathrm{NCs}-$ where, reference samples are also tested for comparison.

\section{Conclusions}

In summary, a new synthesis method has been demonstrated for fully inorganic and hybrid organicinorganic lead bromide perovskite NCs without the use of amines or carboxylic acids. Alternatively, a phosphine-based ligand combination has been employed that allowed dissolution of all the precursors at RT using industrially friendly solvents such as octadecene. Compared to the LARP method, this approach does not require the use of polar solvents resolving the difficulties related to their implementation. The results revealed that light emission characteristics of NCs were superior when the synthesis was performed at RT as opposed to the hot-injection approach. In addition, ligand interactions to the NCs core have been investigated and shown that the Pb-O-P bond was formed on the surface of NCs. Such surface passivation showed to be easier to remove after the antisolvent treatment, resulting in lower organic content of purified NCs as opposed to traditionally used ligands. The new synthesis method provides a simple strategy to produce perovskite NCs with several advantages over the traditional hot-injection or RT route.

\section{Acknowledgements}

The authors acknowledge financial support from Surface Measurement Systems Ltd. T.J.M would like to thank the Ramsay Memorial Trust for their financial assistance. T.J.M and I.P.P would like to acknowledge the EPSRC for financial support (EP/M015157/1). 


\section{References}

[1] M. M. Lee, J. Teuscher, T. Miyasaka, T. N. Murakami, H. J. Snaith, Science 2012, 338, 643647.

[2] H. L. Wells, Z. Für Anorg. Chem. 1893, 3, 195-210.

[3] M. Liu, M. B. Johnston, H. J. Snaith, Nature 2013, 501, 395-398.

[4] A. Kojima, K. Teshima, Y. Shirai, T. Miyasaka, J. Am. Chem. Soc. 2009, 131, 6050-6051.

[5] T. J. Macdonald, M. Batmunkh, C.-T. Lin, J. Kim, D. D. Tune, F. Ambroz, X. Li, S. Xu, C. Sol, I. Papakonstantinou, et al., Small Methods n.d., 0, 1900164.

[6] S. Jeon, L. Zhao, Y.-J. Jung, J. W. Kim, S.-Y. Kim, H. Kang, J.-H. Jeong, B. P. Rand, J.-H. Lee, Small 2019, 15, 1900135.

[7] G. Cen, Y. Liu, C. Zhao, G. Wang, Y. Fu, G. Yan, Y. Yuan, C. Su, Z. Zhao, W. Mai, Small 2019, 15, 1902135.

[8] N. Vicente, G. Garcia-Belmonte, J. Phys. Chem. Lett. 2017, 8, 1371-1374.

[9] J. Shamsi, A. S. Urban, M. Imran, L. De Trizio, L. Manna, Chem. Rev. 2019, 119, 3296-3348.

[10] M. Abdi-Jalebi, Z. Andaji-Garmaroudi, S. Cacovich, C. Stavrakas, B. Philippe, J. M. Richter, M. Alsari, E. P. Booker, E. M. Hutter, A. J. Pearson, et al., Nature 2018, 555, 497-501.

[11] Q. Shan, J. Song, Y. Zou, J. Li, L. Xu, J. Xue, Y. Dong, B. Han, J. Chen, H. Zeng, Small 2017, $13,1701770$.

[12] L. C. Schmidt, A. Pertegás, S. González-Carrero, O. Malinkiewicz, S. Agouram, G. Mínguez Espallargas, H. J. Bolink, R. E. Galian, J. Pérez-Prieto, J. Am. Chem. Soc. 2014, 136, 850-853.

[13] L. Protesescu, S. Yakunin, M. I. Bodnarchuk, F. Krieg, R. Caputo, C. H. Hendon, R. X. Yang, A. Walsh, M. V. Kovalenko, Nano Lett. 2015, 15, 3692-3696.

[14] S. E. Creutz, E. N. Crites, M. C. De Siena, D. R. Gamelin, Chem. Mater. 2018, 30, 4887-4891.

[15] M. Li, R. Begum, J. Fu, Q. Xu, T. M. Koh, S. A. Veldhuis, M. Grätzel, N. Mathews, S. Mhaisalkar, T. C. Sum, Nat. Commun. 2018, 9, 1-7.

[16] A. Swarnkar, A. R. Marshall, E. M. Sanehira, B. D. Chernomordik, D. T. Moore, J. A. Christians, T. Chakrabarti, J. M. Luther, Science 2016, 354, 92-95.

[17] H. Huang, M. I. Bodnarchuk, S. V. Kershaw, M. V. Kovalenko, A. L. Rogach, ACS Energy Lett. 2017, 2, 2071-2083.

[18] S. Wei, Y. Yang, X. Kang, L. Wang, L. Huang, D. Pan, Chem. Commun. 2016, 52, 7265-7268.

[19] G. Almeida, O. J. Ashton, L. Goldoni, D. Maggioni, U. Petralanda, N. Mishra, Q. A. Akkerman, I. Infante, H. J. Snaith, L. Manna, J. Am. Chem. Soc. 2018, 140, 14878-14886.

[20] F. Zhang, S. Huang, P. Wang, X. Chen, S. Zhao, Y. Dong, H. Zhong, Chem. Mater. 2017, 29, 3793-3799.

[21] F. Zhang, H. Zhong, C. Chen, X. Wu, X. Hu, H. Huang, J. Han, B. Zou, Y. Dong, ACS Nano 2015, 9, 4533-4542.

[22] X. Li, D. Yu, F. Cao, Y. Gu, Y. Wei, Y. Wu, J. Song, H. Zeng, Adv. Funct. Mater. 2016, 26, 5903-5912.

[23] O. Vybornyi, S. Yakunin, M. V. Kovalenko, Nanoscale 2016, 8, 6278-6283.

[24] L. Rao, Y. Tang, C. Song, K. Xu, E. T. Vickers, S. Bonabi Naghadeh, X. Ding, Z. Li, J. Z. Zhang, Chem. Mater. 2019, 31, 365-375.

[25] D. Yu, F. Cao, Y. Gao, Y. Xiong, H. Zeng, Adv. Funct. Mater. 2018, 28, 1800248.

[26] E. Yassitepe, Z. Yang, O. Voznyy, Y. Kim, G. Walters, J. A. Castañeda, P. Kanjanaboos, M. Yuan, X. Gong, F. Fan, et al., Adv. Funct. Mater. 2016, 26, 8757-8763.

[27] Y. Tan, Y. Zou, L. Wu, Q. Huang, D. Yang, M. Chen, M. Ban, C. Wu, T. Wu, S. Bai, et al., ACS Appl. Mater. Interfaces 2018, 10, 3784-3792.

[28] D. J. Norris, M. G. Bawendi, Phys. Rev. B 1996, 53, 16338-16346.

[29] L. Protesescu, S. Yakunin, M. I. Bodnarchuk, F. Krieg, R. Caputo, C. H. Hendon, R. X. Yang, A. Walsh, M. V. Kovalenko, Nano Lett. 2015, 15, 3692-3696.

[30] L. Protesescu, S. Yakunin, M. I. Bodnarchuk, F. Bertolotti, N. Masciocchi, A. Guagliardi, M. V. Kovalenko, J. Am. Chem. Soc. 2016, 138, 14202-14205.

[31] A. I. Ekimov, F. Hache, M. C. Schanne-Klein, D. Ricard, C. Flytzanis, I. A. Kudryavtsev, T. V. Yazeva, A. V. Rodina, A. L. Efros, JOSA B 1993, 10, 100-107.

[32] O. Chen, J. Zhao, V. P. Chauhan, J. Cui, C. Wong, D. K. Harris, H. Wei, H.-S. Han, D. Fukumura, R. K. Jain, et al., Nat. Mater. 2013, 12, 445-451. 
[33] R. Zhu, Z. Luo, H. Chen, Y. Dong, S.-T. Wu, Opt. Express 2015, 23, 23680-23693.

[34] Q. A. Akkerman, L. Martínez-Sarti, L. Goldoni, M. Imran, D. Baranov, H. J. Bolink, F. Palazon, L. Manna, Chem. Mater. 2018, 30, 6915-6921.

[35] H. R. Moon, J. J. Urban, D. J. Milliron, Angew. Chem. Int. Ed. 2009, 48, 6278-6281.

[36] V. K. Ravi, A. Swarnkar, R. Chakraborty, A. Nag, Nanotechnology 2016, 27, 325708.

[37] D. N. Minh, J. Kim, J. Hyon, J. H. Sim, H. H. Sowlih, C. Seo, J. Nam, S. Eom, S. Suk, S. Lee, et al., Chem. Mater. 2017, 29, 5713-5719.

[38] B. Tian, B. Tian, B. Smith, M. C. Scott, Q. Lei, R. Hua, Y. Tian, Y. Liu, Proc. Natl. Acad. Sci. 2018, 115, 4345-4350.

[39] H. S. Liu, T. S. Chin, S. W. Yung, Mater. Chem. Phys. 1997, 50, 1-10.

[40] A. G. Young, N. Al-Salim, D. P. Green, A. J. McQuillan, Langmuir 2008, 24, 3841-3849.

[41] J. K. Lorenz, A. B. Ellis, J. Am. Chem. Soc. 1998, 120, 10970-10975.

[42] T. Xuan, X. Yang, S. Lou, J. Huang, Y. Liu, J. Yu, H. Li, K.-L. Wong, C. Wang, J. Wang, Nanoscale 2017, 9, 15286-15290.

[43] N. Al-Salim, A. G. Young, R. D. Tilley, A. J. McQuillan, J. Xia, Chem. Mater. 2007, 19, 51855193.

[44] J. E. B. Katari, V. L. Colvin, A. P. Alivisatos, J. Phys. Chem. 1994, 98, 4109-4117.

[45] J. R. D. la Rosa, P. Elizondo-Martínez, B. N. Martínez, N. P. Rodríguez, J. R. Díaz, Mater. Manuf. Process. 2011, 26, 304-310.

[46] L. M. Wheeler, E. M. Sanehira, A. R. Marshall, P. Schulz, M. Suri, N. C. Anderson, J. A. Christians, D. Nordlund, D. Sokaras, T. Kroll, et al., J. Am. Chem. Soc. 2018, 140, 1050410513. 